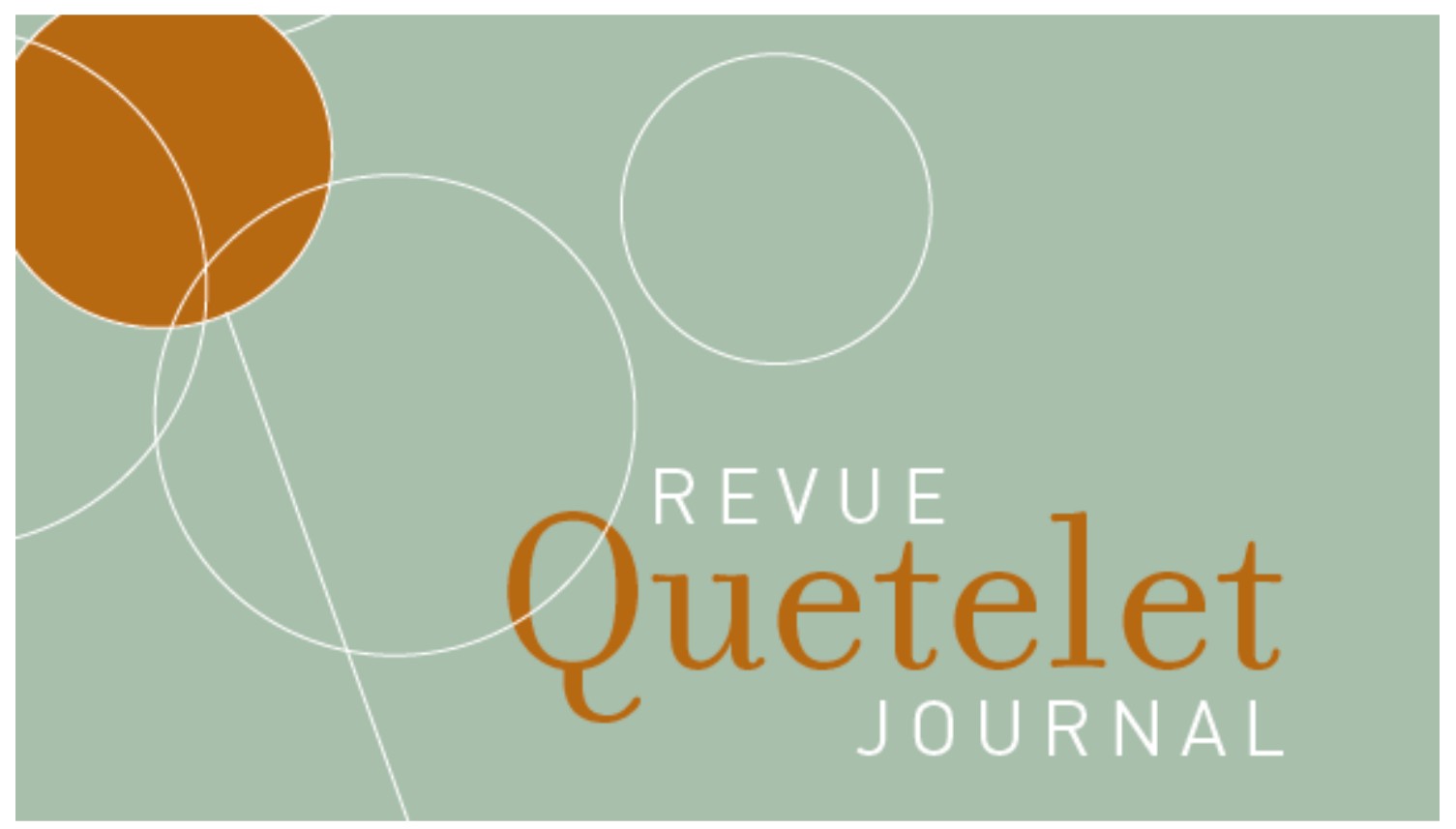

Vol. 4, n 1, avril 2016, pp. 101-121

DOI : 10.14428/rqj2016.04.01.05

ISSN: 20349378

De la procréation "assistée par» autrui à la procréation "négociée avec» autrui : dialogue autour de la place du tiers donneur dans le projet parental de mères lesbiennes au Québec

Isabel Côté, Kévin Lavoie

C2016 Isabel Côté, Kévin Lavoie

This work is licensed under a Creative Commons Attribution-NonCommercial 4.0 International License. You can share, adapt the material for non-commercial purposes provided that you give appropriate credit and indicate if changes were made. For details see https://creativecommons.org/licenses/by-nc/4.0/

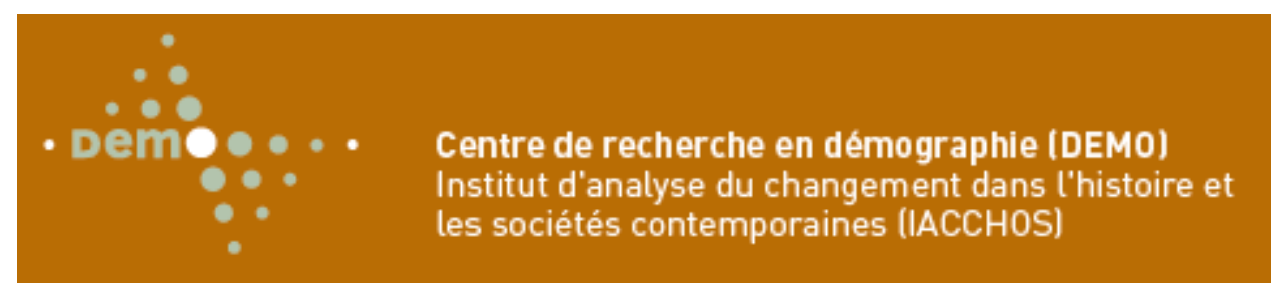




\title{
De la procréation «assistée par» autrui à la procréation «négociée avec» autrui : dialogue autour de la place du tiers donneur dans le projet parental de mères lesbiennes au Québec
}

\author{
ISABEL CÔTÉ ${ }^{1}$ \\ KÉVIN LAVOIE ${ }^{2}$
}

\begin{abstract}
Résumé
Cet article présente le cadre de négociation concernant la place des hommes qui participent au projet parental de mères lesbiennes en tant que donneurs connus au Québec. Trente-deux personnes ont été rencontrées, soit neuf couples de femmes et dix donneurs, de même que quatre partenaires de vie de ces hommes. Les résultats démontrent que le don s'inscrit dans une démarche négociée, à la jonction de trois considérations désignées comme des facteurs d'influence : les réflexions préalables des mères quant à leurs représentations de la paternité et de l'engagement désiré de la part du donneur, les attentes singulières qui motivent ces hommes à participer de façon consentante au projet parental d'autrui et, dans certains cas, la façon dont leur partenaire appréhende les impacts potentiels de ce don sur leur vie conjugale et familiale. Cette négociation à trois ou à quatre croise aussi les préoccupations qu'ont ces adultes à l'égard des besoins des enfants, formant ainsi une toile complexe de relations interpersonnelles. Le dialogue semble être le garant de la cohésion entre les personnes concernées et favorise l'émergence chez le donneur d'un rôle en regard de l'enfant qui soit conforme aux attentes de toutes les parties.
\end{abstract}

\footnotetext{
1. Département de travail social, Université du Québec en Outaouais, Canada.

2. Faculté des arts et sciences, Université de Montréal, Canada.
} 
102 De la procréation «assistée par» autrui à la procréation «négociée avec» autrui : dialogue autour de la place du tiers donneur dans le projet parental de mères lesbiennes au Québec

\title{
Mots-clés
}

Homoparenté, don de sperme, procréation assistée par autrui, négociation, Canada, Québec.

\begin{abstract}
This paper presents the negotiation framework concerning the place of men involved in lesbian mother's parental project as known donors in Quebec. Thirty-two people were interviewed, nine women couples and ten donors, as well as four partners of these men. The results show that the donation is part of a negotiated approach, at the junction on three considerations identified as influencing factors: prior thoughts of mothers about their perceptions of fatherhood and the required commitment from the donor, singular expectations driving these men to participate in a consented way to the parental project of others and, in some cases, how their partner grasps the potential impacts of this gift on family and conjugality life. This negotiation also crossed concerns expressed by these adults towards children's needs, forming a complex web of relationships. The dialogue seems to be the guarantor of cohesion between the people involved, while facilitating the emergence in the donor's role towards the child that meets the expectations of them all.
\end{abstract}

\section{Keywords}

Same-sex kinship, sperm donation, assisted human reproduction, negociation, Canada, Quebec.

\section{Introduction}

Les familles homoparentales font l'objet de recherches en sciences humaines et sociales depuis déjà quatre décennies et occupent une place grandissante dans l'espace public des sociétés occidentales (Gross, 2015). Ces familles regroupent des configurations familiales diversifiées, dont celles faisant l'objet du présent article : les familles lesboparentales dont les enfants sont nés d'un donneur connu. Par «donneur connu», il est généralement entendu un homme acceptant d'agir à titre de tiers reproducteur pour le projet parental d'autrui en contexte privé (Côté et al., 2015). Il peut alors s'agir d'un membre de l'entourage du couple lesbien ou d'un homme contacté par le biais de sites Web. Ce type de donneur se distingue d'un donneur anonyme ayant offert ses gamètes à une banque de sperme, et ce, même si certains d'entre eux acceptent que des informa- 
tions les concernant soient éventuellement accessibles aux enfants qui seront issus de leurs dons ${ }^{3}$.

Plusieurs études ont été conduites au fil des années pour mieux comprendre la réflexion qui sous-tend le recours à un donneur connu pour concrétiser un projet familial plutôt que d'opter pour un donneur via les banques de sperme (Côté, 2012 ; Kelly, 2009 ; Norsqvist, 2012 ; Ryan, Berkowitz, 2009; Svab, 2007). Outre les difficultés d'accès aux cliniques de fertilité et les coûts associés aux techniques de reproduction assistée, les motivations des mères lesbiennes d'établir leur famille avec l'aide d'un donneur connu sont liées à l'importance qu'elles accordent à l'aspect biogénétique comme fondement identitaire de leur progéniture et le désir de socialiser leurs enfants auprès du donneur, qu'il soit ou non reconnu comme leur père. Ces pratiques s'inscrivent dans un contexte sociétal où l'importance des liens génétiques dans le «faire famille» et l'engagement du père pour le développement de l'enfant nourrissent certaines trames discursives sur la famille (Descoutures, 2010 ; Ryan, Berkowitz, 2009; Svab, 2007).

D'autres recherches, beaucoup moins nombreuses toutefois, ont documenté les motivations des donneurs de sperme à participer au projet parental d'autrui. Pour certains de ces hommes, le don de sperme représente une façon d'avoir des enfants, mais sans en avoir réellement (Almeling, 2011; Côté, 2012, 2014 ; Daniels et al., 2012). Riggs (2008) parle alors d'un «désir d'immortalité génétique» plutôt que d'un désir de paternité. Être un père biologique, mais sans assumer de rôle parental ou être impliqué dans les activités quotidiennes à titre de papa peut être suffisant pour combler le désir d'enfants de certains donneurs. Malgré leur faible investissement dans l'exercice de la parentalité, plusieurs de ces hommes ressentent une connexion affective avec les enfants nés de leur don et sont subjugués par les ressemblances physiques ou de caractère qu'ils remarquent avec eux, considérant ces enfants comme faisant partie de leur lignée (Almeling, 2011 ; Côté, 2014 ; Dempsey, 2012).

Les mères lesbiennes qui optent pour un donneur connu vont souvent choisir un homme qui partage avec elles plusieurs affinités, notamment sur le plan socioculturel (Mamo, 2007). La relation qu'elles entretiennent avec lui est caractérisée à la fois par un sentiment de confiance et une reconnaissance à l'égard de son don, mais aussi par une certaine mise à

3. Au Canada, l'anonymat des donneurs et donneuses de gamètes prévaut, en vertu de la Loi canadienne sur la procréation assistée (Gouvernement du Canada, 2004). L'enfant né d'un donneur canadien n'aura donc jamais accès à des données nominatives concernant l'homme à l'origine de sa conception, seules des informations de nature médicale pouvant être transmises aux autorités compétentes le cas échéant. 
distance, ceci s'expliquant par la crainte des mères que le donneur s'investisse trop fortement auprès de leur enfant (Nordqvist, 2012). De fait, contrairement aux couples lesbiens qui choisissent d'avoir leurs enfants à l'aide d'un don de sperme en clinique de fertilité, celles qui préfèrent le recours à un donneur connu doivent réfléchir au type de relation qu'elles souhaitent ou non voir s'installer entre ce dernier et leurs enfants (Kelly, 2009). Pour minimiser les risques de conflits, les couples lesbiens et leur donneur discutent de leurs motivations respectives et s'entendent préalablement avant la conception sur le rôle que cet homme endossera ou non dans la vie des enfants (Dempsey, 2012 ; Surtees, 2011). Or, cela est d'autant plus difficile à faire qu'il existe de nombreux types d'arrangements en ce qui concerne les dons entre particuliers (Norsqvist, Smart, 2014). Dans un tel contexte, la volonté de reconnaissance légale de la mère non statutaire et la revendication des droits du donneur en tant que père biologique peuvent engendrer des conflits sur le plan juridique (Descoutures, 2010 ; Millbank, 2008 ; Nordqvist, Smart, 2014).

De leur côté, les hommes doivent aussi prendre le temps de réfléchir à leurs aspirations à l'égard du projet parental du couple lesbien et aux impacts que le don pourrait avoir sur eux, ainsi que sur leurs proches et sur les propres enfants. De fait, les hommes qui ont peu réfléchi de prime abord aux répercussions potentielles du don sur leur équilibre psychologique et émotif sont plus susceptibles d'éprouver des difficultés à se conformer au rôle attendu par les mères auprès de l'enfant (Riggs, 2008). Qui plus est, les couples lesbiens et les hommes qu'elles sollicitent pour les aider à fonder leur famille n'ont pas toujours le réflexe de discuter de l'ensemble des considérations entourant le don ni à se projeter dans l'avenir (Dempsey, 2010 ; Kelly, 2009 ; Surtees, 2011). Passée sous silence lors des discussions de départ, l'inadéquation entre les aspirations du donneur et celles des futures mères peut alors être une source de frustration et un précurseur de conflits (Riggs, 2008).

Dans le contexte québécois, les balises juridiques qu'instaure la Loi instituant l'union civile et les nouvelles règles de filiation (Loi 84) permettent néanmoins de lever l'inquiétude qu'un donneur soit reconnu père d'un enfant né de ses dons au détriment de la mère non biologique. Depuis, 2002, cette Loi permet à deux femmes d'être reconnues légalement sur l'acte de naissance de leur enfant, sans que la mère n'ayant pas porté l'enfant n'ait à s'astreindre à un processus d'adoption (Gouvernement du Québec, 2002). Cette filiation monosexuée d'origine octroie les mêmes droits et responsabilités aux parents de même sexe qu'aux couples formés d'une femme et d'un homme (Bureau, 2009). Pour concrétiser leur projet parental, un couple lesbien québécois peut recourir aux servi- 
ces de cliniques de fertilité ou encore, bénéficier d'un don de sperme en contexte privé d'un homme qui fera office de tiers donneur (Côté et al., 2015). La Loi 84 prévoit que le don de sperme entre particuliers n'engage aucune responsabilité du donneur ni ne lui confère de droit par rapport à l'enfant né de son don4. De fait, l'article 538.2 du Code civil du Québec spécifie que : «L'apport de forces génétiques au projet parental d'autrui ne peut fonder aucun lien de filiation entre l'auteur de l'apport et l'enfant qui en est issu». Même si le statut parental de la mère qui n'a pas porté l'enfant ne peut être contesté au profit du donneur, le couple doit nécessairement entreprendre une réflexion sur la dynamique familiale qu'il souhaite instaurer avant d'entreprendre quelque démarche que ce soit auprès d'un donneur potentiel. Malgré les dispositions de la Loi québécoise venant sécuriser le lien de filiation entre les deux mères et leur enfant, des mésententes peuvent survenir entre les protagonistes. Dans ce contexte, comment s'actualise la dynamique relationnelle entre ces hommes et les couples lesbiens bénéficiaires de leur don?

Dans le présent article, une attention particulière est portée sur la négociation de l'entente de procréation, en croisant les points de vue des mères avec ceux des donneurs et de leur partenaire de vie afin d'en dégager un portrait global, peu exploré dans la littérature jusqu'à maintenant. De fait, les recherches portant sur le sujet tendent à documenter le point de vue des mères ou des donneurs séparément, alors que celui des partenaires de vie des donneurs est occulté. La juxtaposition des points de vue de l'ensemble des personnes impliquées constitue donc un aspect original de la présente étude, laquelle aborde deux éléments charnières permettant de mieux comprendre la dynamique relationnelle entre les mères lesbiennes, les donneurs et leur partenaire de vie. D'abord, les facteurs venant soutenir et influencer la dynamique sont présentés à l'aune des expériences croisées des personnes impliquées. Ensuite, les éléments faisant l'objet d'une négociation sont considérés, situant alors la démarche de procréation dans une approche relationnelle. Le dialogue semble être le garant de la cohésion familiale et du respect de l'entente initiale, favorisant par le fait même l'émergence chez le donneur d'un rôle en regard de l'enfant qui soit conforme aux attentes de toutes les parties.

4. À moins que le don ne résulte d'une relation sexuelle entre le donneur et la mère biologique, auquel cas le donneur a un an à partir de la naissance de l'enfant pour réclamer sa paternité s'il le souhaite (art. 538.2 C.c.Q.). Notons que depuis la mise en œuvre de la Loi, aucune réclamation en paternité en vertu de cet article n'a été entreprise en justice. Pour un complément d'information, voir Côté, Lavoie et de Montigny, (2015). 
106 De la procréation «assistée par» autrui à la procréation «négociée avec» autrui : dialogue autour de la place du tiers donneur dans le projet parental de mères lesbiennes au Québec

\section{Méthodologie}

Cette étude qualitative ${ }^{5}$ s'appuie sur deux collectes de données. La première s'est déroulée d'avril 2010 à janvier 2011 et visait à appréhender le rôle du donneur auprès des enfants nés de ses dons (Côté, 2012). Neuf triades ont été rencontrées à cette époque $(n=27)$, soit neuf couples de femmes et leur donneur respectif. Les résultats ont démontré des niveaux différenciés d'implication du donneur auprès des enfants (Côté, 2014). La mise à distance ou l'appropriation par les mères d'une représentation exclusive de la filiation s'est révélée être un facteur déterminant. Ainsi, une forte adhésion au modèle nucléaire et une minimisation de l'importance du père comme figure parentale éloignent le donneur de la cellule familiale, tandis que l'ouverture à la pluriparentalité et l'importance accordée à la présence d'une figure paternelle de la part des mères sont deux facteurs qui contribuent à favoriser la présence du donneur dans la vie des enfants. Quant aux donneurs, leur rôle est fortement imprégné de leurs attentes et de leurs motivations initiales à participer au projet parental du couple lesbien avec lequel ils entretiennent, pour la plupart d'entre eux, un rapport relationnel en tant qu'ami, frère de la mère n'ayant pas porté l'enfant ou collègue de travail (Côté, 2012).

Les femmes et les hommes ont été à nouveau sollicités à l'automne 2013 afin de voir dans quelle mesure le rôle du donneur était susceptible de se transformer, notamment en regard de l'évolution des relations nouées entre les protagonistes et les sollicitations potentielles des enfants issus des dons. Dans une situation, le portrait familial s'était modifié par la naissance d'un troisième enfant issu d'un donneur différent de ceux des aînés. Lors de cette deuxième collecte de données, la chercheure responsable du projet a voulu donner une voix à un membre jusque-là invisible, mais néanmoins essentiel, de la dynamique triadique instaurée entre les couples et leur donneur, c'est-à-dire le conjoint ou la conjointe de ce dernier. Quatre partenaires de vie de ces donneurs, un homme et trois femmes, ont donc aussi été rencontrés à cette occasion, pour un total de trente-deux $(n=32)$ participantes et participants.

Plusieurs stratégies ont été déployées pour constituer l'échantillon de départ. Différentes organisations québécoises vouées à la diversité sexuelle ont d'abord été approchées afin qu'elles sollicitent leurs membres correspondant au profil recherché. Les médias sociaux tel Facebook ont aussi

5. Cette étude a été rendue possible grâce à l'appui du Conseil de recherches en sciences humaines du Canada (CRSH) et du Fonds de recherche du Québec - Société et culture (FRQSC). 
été utiles pour diffuser l'appel à participation. La méthode «boule-de-neige» a permis de compléter notre échantillon (Pires, 1997). Sur le plan sociodémographique, les dix-huit mères lesbiennes ont en moyenne 38 ans, tandis que les dix donneurs sont âgés en moyenne de 40 ans au moment de la deuxième vague d'entrevues. Cinq d'entre eux s'identifient comme homosexuels, tandis que les autres sont d'orientation hétérosexuelle. Cinq étaient en couple au moment de l'entretien, les autres étaient célibataires. Plusieurs donneurs ont contribué à la naissance de plus d'un enfant : quatre hommes ont fait des dons pour un enfant, cinq en ont fait pour deux enfants et un autre a vu trois enfants naître de ses dons. Tous les enfants sont nés après 2002, année de mise en œuvre de la Loi 84. Enfin, il s'agit d'une population possédant un niveau d'éducation élevé. Onze mères possèdent un diplôme universitaire, cinq autres ont terminé une formation collégiale et six étaient aux études au moment des entretiens. Quatre participantes sont titulaires d'un doctorat ou d'un postdoctorat. Les donneurs ne sont pas en reste, alors que huit détiennent un diplôme universitaire et les deux autres ont fait des études collégiales. Parmi ceux ayant poursuivi des études universitaires, deux étaient titulaires d'un doctorat. Quant aux quatre partenaires des donneurs rencontrés, il et elles détiennent tous au moins un diplôme universitaire de premier cycle.

Le corpus de données a été recueilli à l'aide d'entrevues semi-dirigées (Savoie-Zajc, 2010). Au cours de ces entrevues, plusieurs thèmes ont été abordés, soit les motivations des participantes à réaliser leur rêve d'enfant avec un donneur connu et celles de ces hommes à participer au projet parental d'autrui, leurs représentations respectives des figures paternelles et maternelles, ainsi que l'impact de ces représentations sur la dynamique relationnelle, notamment en ce qui à trait à la négociation de l'entente de procréation assistée. Les entrevues individuelles ont par la suite fait l'objet d'une analyse de contenu thématique (Bardin, 2007) inspirée de la théorie du parcours de vie (Elder et al., 2004). Dans le contexte de la présente recherche, ce cadre d'analyse s'est révélé particulièrement fécond puisqu'il offre un appareil conceptuel articulé et dynamique permettant de mieux comprendre la complexité des liens existants entre le temps, les trajectoires sociales, le développement individuel, les relations interpersonnelles et les contextes sociohistoriques. Le parcours de vie est ponctué par des événements et des transitions qui représentent des périodes de courte durée et indiquent un changement de statut ou de rôle. Les événements jalonnant la vie des mères et des donneurs ont été analysés dans l'optique de mieux comprendre leur concordance sur la dynamique relationnelle des personnes concernées. Les résultats présentés ici ne visent donc pas à faire état de l'évolution du rôle du donneur dans la vie des enfants, mais bien à situer les éléments du cadre de négociation 
108 De la procréation «assistée par» autrui à la procréation «négociée avec» autrui : dialogue autour de la place du tiers donneur dans le projet parental de mères lesbiennes au Québec

faisant en sorte que la dynamique relationnelle entre les mères et le donneur, et son ou sa partenaire de vie le cas échéant, semble exempte de conflits.

Enfin, il importe de savoir que la communauté gaie et lesbienne est une petite communauté au Québec et que les familles correspondant à notre objet d'étude sont peu nombreuses. La confidentialité et l'impossibilité de traçabilité des personnes interrogées revêtent donc, dans la situation présente, une importance primordiale. C'est pourquoi notre certification éthique est basée sur le respect scrupuleux de leur identification et, conséquemment, nous ne situerons, dans les extraits, que la position du répondant ou de la répondante dans l'unité familiale. Par ailleurs, puisque nous sommes engagés à éviter l'identification croisée des participants entre eux, les extraits des membres des différentes unités familiales ne seront pas reliés les uns aux autres.

Les résultats sont présentés dans les sections suivantes. Plusieurs participantes et participants ont mentionné être en train de «construire une nouvelle dynamique familiale» sans avoir ni balises ni modèles sur lesquels s'appuyer, la procréation à l'aide d'un tiers connu étant une pratique marginale et peu documentée, tant sur le plan scientifique que dans les milieux sociosanitaires et associatifs. Chacune des familles a donc développé sa propre conception et actualisation du rôle de donneur. La négociation apparaît comme un moment charnière et déterminant dans le maintien d'une saine dynamique relationnelle entre les personnes impliquées, tant au regard des facteurs d'influence de chaque groupe que des éléments considérés pour l'établissement de l'entente de don.

\section{Les facteurs d'influence comme prémisses à la négociation}

Plusieurs facteurs d'influence sont à l'œuvre avant et pendant la réalisation du projet lesboparental à l'aide d'un donneur connu (Côté, 2012). Ces facteurs, bien qu'ils soient spécifiques à chacun des groupes concernés - mères lesbiennes, donneurs et les partenaires de vie de ces hommes -, s'entrecroisent pour déterminer l'issue de la négociation, laquelle vise à circonscrire le rôle que jouera le donneur dans la vie des enfants issus de ses dons. La figure suivante modélise le dialogue entre les adultes concernés par l'entente de procréation, au sein duquel les facteurs d'influence des trois acteurs posent les prémisses à la négociation impliquant six considérations relationnelles. Chacun de ses éléments est décortiqué dans les prochaines sections. 
FIGURE 1 Modèle de négociation entourant le rôle du tiers donneur

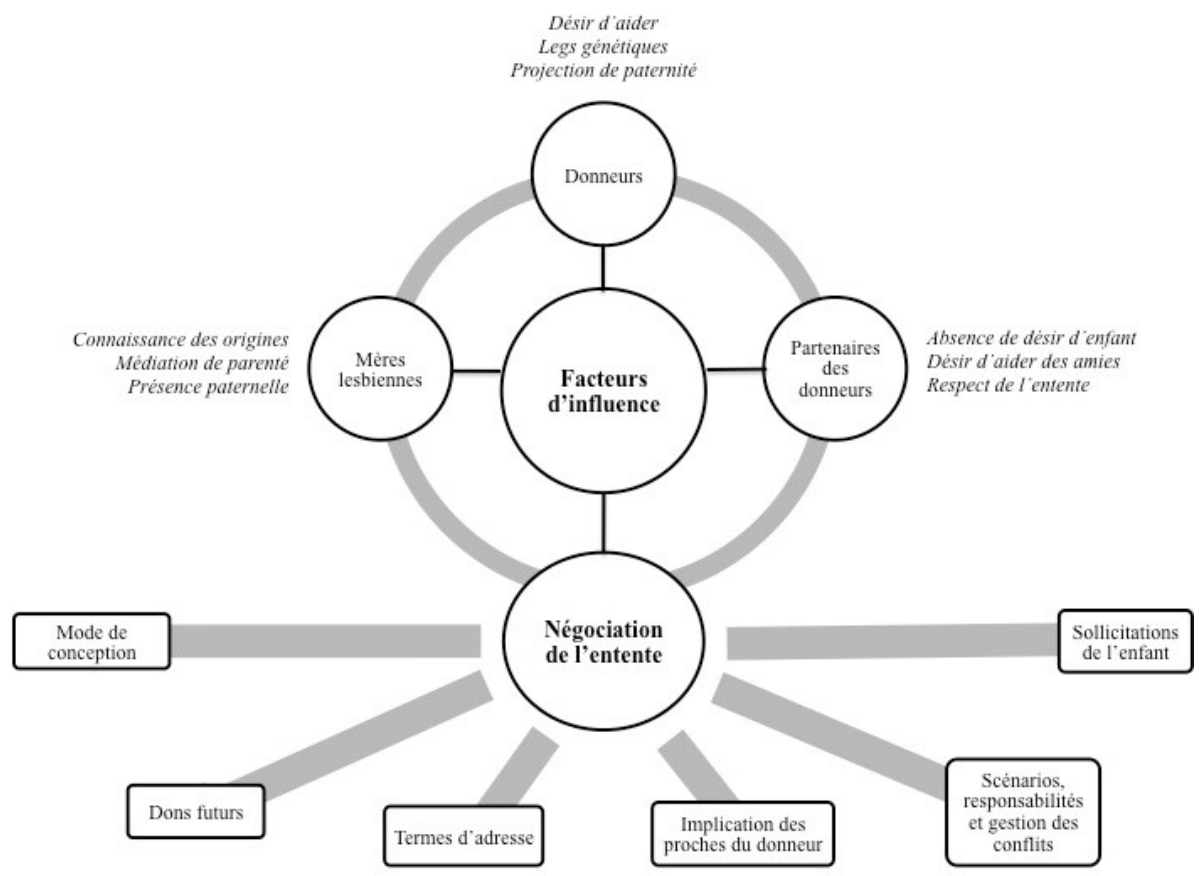

Facteurs d'influence des mères

Les facteurs ayant influencé les mères lesbiennes rencontrées à opter pour un donneur connu pour concrétiser leur projet parental se répartissent en quatre éléments. D'abord, l'importance que les mères accordent à la connaissance des origines est un facteur déterminant dans leur choix de concrétiser leur projet parental à l'aide d'un donneur connu. Toutes les femmes ont en effet témoigné de leurs réticences à fonder leur famille à l'aide d'un donneur anonyme, comme c'est le cas actuellement dans les cliniques de fertilité au Québec. Le désir d'intégrer le don dans l'histoire familiale et personnelle de leur enfant est saillant chez toutes les femmes rencontrées, de même que la possibilité de lui faire rencontrer un jour son géniteur.

Ensuite, pour cinq couples rencontrés, l'établissement d'une filiation biologique entre les enfants du couple par l'entremise de l'apport du même donneur est une stratégie de médiation de parenté, c'est-à-dire une façon de créer des liens entre les membres de la famille. Cela est d'autant plus important lorsque chacune des mères envisage de porter un enfant. À leurs yeux, ce lien génétique a le mérite de souder la relation fraternelle. 
110 De la procréation «assistée par» autrui à la procréation «négociée avec» autrui : dialogue autour de la place du tiers donneur dans le projet parental de mères lesbiennes au Québec

De même, le souhait de partage du génotype entre la mère n'ayant pas porté ses enfants et sa progéniture peut amener le couple à considérer un membre de la famille de la mère sociale comme donneur potentiel, comme c'est justement le cas d'une famille de l'échantillon.

Enfin, le recours à un donneur connu évoque la présence plus ou moins soutenue d'une figure paternelle auprès des enfants. En effet, les mères concernées sont sensibles au discours sur l'engagement et la présence d'un père, et de leurs bienfaits sur le développement infantile. Elles ont longuement réfléchi à la question de l'identité du donneur et à son statut au sein de leur famille. Deux couples ont ainsi choisi le donneur à condition qu'il accepte de s'engager de façon significative dans un rapport filial et affectif avec les enfants et qu'il soit connu d'eux comme leur père. Elles se considèrent néanmoins comme les seuls parents légitimes et ne souhaitent pas partager le parentage avec une troisième personne, fût-il le père biologique de l'enfant. Cette paternité est alors symbolique.

\section{Facteurs d'influence des donneurs}

Les facteurs ayant mené les donneurs à accepter une demande ou à offrir leur aide à un couple lesbien proviennent de trois types d'influence. Pour quatre donneurs, la principale motivation était de venir en aide à des amies. Le don est alors un geste désintéressé visant à les aider à concrétiser leur rêve de fonder une famille.

Trois hommes évoquent plutôt leur intérêt à offrir leurs gamètes pour «laisser une trace», ce qui renvoie à l'idée de legs génétiques. Ces donneurs se disent flattés que leurs gènes soient désirés. Pour un autre donneur, ce don fut, en quelque sorte, un compromis lui permettant d'avoir un enfant lui étant génétiquement lié, malgré l'absence de descendance.

«À la base, c'est certain que j'ai toujours voulu avoir des enfants. Ma conjointe
n'est pas chaude à l'idée. Elle ne veut pas d'enfant. Pour moi, c'était le désir de
me voir dans quelqu'un d'autre. Je ne sais pas exactement comment l'expliquer.
En fait, c'était important pour moi de continuer mes gènes». (Donneur)

L'implication dans le projet lesboparental instille le désir de certains hommes d'investir une identité paternelle, généralement de façon symbolique. Hormis deux hommes, aucun d'entre eux ne souhaitait investir le champ parental. C'est d'ailleurs en l'absence de responsabilités liées au parentage qu'ils ont accepté de jouer ce rôle. Malgré tout, le don est perçu par tous les donneurs comme une expérience valorisante. À ce propos, trois des donneurs gais avaient un désir de paternité depuis l'enfance, mais leur homosexualité a longtemps été considérée comme un obstacle 
à la réalisation de leur rêve. La demande du couple lesbien a alors été considérée comme une opportunité.

«J'ai toujours voulu avoir des enfants. J'ai toujours voulu être père. Mais parce que je suis gai, je ne savais pas comment je pouvais devenir père». (Donneur)

\section{Facteurs d'influence des partenaires de vie des donneurs}

Peu documentée, la voix des partenaires de vie des donneurs connus offre pourtant un éclairage nouveau sur le processus de procréation à l'aide d'un tiers. Leur présence influence grandement le dialogue entre les personnes concernées, passant d'une négociation à trois à une discussion entre deux couples. L'un des facteurs qui incitent les partenaires à soutenir le projet est leur absence de désir d'enfant, l'implication du conjoint à titre de donneur ne menaçant pas un quelconque projet du couple de fonder leur propre famille.
«Ma copine a été un appui sans aucune réserve, elle a toujours été avec moi là- dedans, elle a toujours été à $100 \%$ d'accord avec tout. Ça aurait été bien plus difficile pour moi si on n'avait pas été totalement en accord là-dessus. Je lui en ai parlé avant qu'on commence à se fréquenter [...], mais je peux bien imaginer que c'est quasiment une décision de couple. [...] Elle avait la peur que je m'attache à l'enfant plus qu'à elle. Tu sais, qu'elle soit délaissée dans tout ça parce qu'elle n'a pas d'enfant et qu'elle n'en veut pas». (Donneur)

Le désir d'aider des amies est un autre facteur invitant les partenaires des donneurs à participer au projet parental d'autrui, en accompagnant leur conjoint dans cette aventure. Cette participante raconte avoir été témoin, ainsi que son conjoint, des démarches effectuées par le couple pour se trouver un donneur pour agrandir leur famille, après que celui des aînés ait choisi de se désister pour la suite des choses. Loin d'adopter un rôle passif, cette dernière joue en quelque sorte un rôle d'entremetteuse entre son couple d'amies et son conjoint.

«On les a accompagnées là-dedans. D'un mois à l'autre, toujours pas de grossesse.
On les a vues se décourager. Elles commençaient à penser à une banque [de sper-
me] puis à se demander 'Est-ce qu'on laisse tomber le projet ?'. Tout ce temps-là,
moi je me disais : 'Mon conjoint ferait un bon donneur'. [...] Je n'osais pas lui en
parler. J'attendais que ça vienne de lui. J'avais commencé à y penser à partir du
moment où elles ont dit que [le premier donneur] ne voulait plus faire de don.
J'ai dit: 'Si c'était toi ? Pourquoi pas ?'». (Partenaire d'un donneur)

Enfin, le respect de l'entente établie entre le donneur et le couple lesbien s'est avéré être un facteur d'influence dans deux situations, ce qui signifie que la partenaire a accepté, généralement au début de sa fréquentation avec son nouveau conjoint, le projet préexistant qui le liait avec les mères. 
«Au début de notre relation, mon conjoint m'a dit : 'Il faut que je te dise quelque chose. Ma sœur est homosexuelle. Puis elles ont un enfant, et un autre qui est en route et c'est moi le donneur. As-tu un problème avec ça ?' [rires] J'ai dit : 'De prime abord, non'. C'est sûr que ça m'a un peu saisie, mais je dirais que ce n'était pas négatif du tout. Non, je trouvais cela très intéressant». (Partenaire d'un donneur)

\title{
La négociation au carrefour de considérations relationnelles
}

La négociation est un moment charnière où convergent les différents facteurs d'influence des parties impliquées. Différents thèmes sont alors discutés entre les protagonistes afin de dessiner une dynamique relationnelle qui rejoigne les attentes de chacune et chacun. Parfois concentrée dans un moment particulier, souvent échelonnée sur plusieurs semaines ou plusieurs mois, la négociation témoigne, non seulement de la souplesse des personnes impliquées et de leurs aspirations, mais également de leur préoccupation à l'endroit des besoins appréhendés de l'enfant à naître. Plusieurs éléments sont ainsi objet de négociation, dont la méthode de conception et les dons futurs, les termes d'adresse utilisés pour désigner le donneur, les responsabilités dévolues au donneur advenant le décès des mères et, finalement, les sollicitations éventuelles de l'enfant envers le donneur.

\section{Mode de conception et dons futurs}

Le premier élément de négociation porte sur le mode de conception retenu par le couple lesbien et le donneur. L'absence de relation sexuelle entre le donneur et la future mère biologique est alors convenue et dans tous les cas, c'est la méthode d'insémination artisanale qui a donc été retenue. Cette négociation prévoit également les responsabilités de chacun advenant d'éventuels problèmes.

\begin{abstract}
«Avant de faire le don, on a eu une bonne discussion sur comment on voyait ça. Elles nous ont fait signer un papier pour formaliser le fait qu'il n'y aurait pas de recours contre mon conjoint pour une pension alimentaire. Puis on a discuté de comment on allait vivre ça les deux familles ensemble, nommer les choses pour ne pas que ça crée de malaise parce que notre amitié est importante». (Partenaire d'un donneur)
\end{abstract}

La période pendant laquelle s'échelonnent les dons est vécue de façon particulière pour certains donneurs. En effet, quatre d'entre eux ont témoigné avoir trouvé cette période spécialement intense, astreignante et temporellement énergivore du fait qu'il leur fallait être disponibles au 
moment de l'ovulation de la future mère. Cela impliquait de quitter le travail plus tôt, de s'y rendre plus tard, de parcourir une certaine distance pour aller à la rencontre du couple, sans compter le stress de «ne pas y arriver». Les conjointes concernées, loin d'être des témoins passifs de ce moment crucial, sont, au contraire, des actrices impliquées dans le processus. Cette implication présente un double avantage. D'une part, cela permet de circonscrire la sexualité du donneur - inhérente au processus - au sein de son couple et d'autre part, permet à la partenaire de faire partie prenante du processus de procréation.

\begin{abstract}
«Le fait que ce soit moi qui amène [le don de sperme], ça me donnait une place dans la démarche. J'avais un rôle, puis ça défaisait le risque qu'il y ait un malaise du fait que mon conjoint donne à mon amie. J'étais l'intermédiaire, j'étais consentante. Symboliquement, ça faisait mon affaire que ce soit moi qui aille le donner. [...] C'est un beau cadeau de vie qu'on a fait. J'en parle comme si c'était moi qui ai fait le cadeau aussi parce qu'on l'a vraiment placé comme si c'était le couple qui donne au couple. J'étais partie prenante de toute la démarche. J'ai été un peu l'initiatrice. Aussi par le fait que je sois impliquée, que je participe à la décision et aux discussions, ça défaisait le malaise. Ça dissociait le don d'une sexualité qui n'avait pas lieu non plus. Oui, ça enlevait le sexe de tout ça». (Partenaire d'un donneur)
\end{abstract}

Quatre donneurs se sont engagés dès le départ à faire des dons pour plus d'un enfant. Deux autres, soupçonnant que tel serait le désir des mères, ont accepté d'emblée lorsque la demande de participer à la naissance d'un deuxième enfant a été formulée quelque temps après la naissance du premier bébé. Enfin, trois donneurs ont affirmé qu'ils accepteraient de faire à nouveau des dons, si le couple concerné le leur demandait. Dans deux cas, les partenaires de vie de deux donneurs étaient présents tout au long de la démarche et ont participé activement au processus menant à la naissance de l'enfant des mères. Dans deux autres situations, la conjointe du donneur s'est jointe à la triade après la naissance d'un ou deux enfants issus des dons. Pour l'une d'elles, l'évolution de la relation de couple a fait alors en sorte que l'entente initiale entre le donneur et le couple lesbien a été renégociée.

«Après le deuxième, c'est là que ça a changé, je dirais, parce qu'elles ont voulu en avoir un troisième. On a accepté les deux premiers parce que ça a été une décision commune de dire oui, mais là, je suis tombée enceinte. On a dit : 'Une fois qu'on aura la nôtre, ce sera fini'. J'ai été hospitalisée deux mois avant d'accoucher, puis [prénom de sa fille] est née, donc ça a été un dossier clos. C'était clair pour nous deux que quand on avait notre propre famille, mon conjoint ne faisait pas de don ailleurs». (Partenaire d'un donneur) 


\title{
Termes d'adresse
}

Toutes les triades ont discuté de leurs attentes et de leur vision quant au niveau désiré d'implication du donneur, la façon dont il serait considéré par rapport à l'enfant et aux mots qui seraient utilisés pour le désigner. Dans le cas des situations où le donneur est en couple, la désignation a une connotation particulière, l'usage des mots «père» et «papa» étant réservé, aux yeux des conjointes, à leurs propres enfants. Ainsi, les partenaires contribuent à définir le rôle du donneur, généralement en le situant à l'extérieur du système de parenté associé à la paternité sociale.

«Je sais que lui, ça ne le dérangerait pas [que les enfants l'appellent Papa]. Mais ce n'est pas leur papa. C'est clair pour moi. Je ne ferais pas une crise pour autant. Je pense qu'on aurait une discussion entre les mères pour le dire. Je pense que c'est leur position aussi [les mères]. Ce n'est pas un père. Ils ont déjà deux parents. Le mot 'papa', je pense qu'il m'énerve [rires]». (Partenaire d'un donneur)

Même en l'absence d'une progéniture, le partenaire du donneur exprime une certaine réserve par rapport à l'utilisation du mot «père» pour désigner le donneur. Il considère en effet que la paternité et les termes qui lui sont associés sont réservés aux hommes qui exercent leur rôle au quotidien, comme les soins aux enfants et l'éducation. L'apport génétique comptant peu à ses yeux, il propose plutôt le terme "oncles spéciaux» pour définir le rôle que les deux membres du couple endossent auprès des enfants, en conformité avec l'entente établie avec les mères.

\begin{abstract}
«On joue le même rôle, mon conjoint et moi. On est là avec les enfants, on a les mêmes relations avec chacun des enfants. Moi, je ne suis pas reconnu comme père d'aucune façon. Je ne vois pas pourquoi il serait reconnu comme père quand tu ne joues pas le rôle, juste parce que c'est lui qui a rempli le petit pot. C'était aussi quelque chose qu'on a abordé directement avec les femmes avant d'avoir les enfants, parce que mon conjoint a demandé s'il aurait le titre de père. [Prénom de la mère sociale] en particulier a dit non parce qu'elle avait une certaine inquiétude par rapport à son rôle vis-à-vis des enfants. Elle était inquiète qu'elle soit moins reconnue comme parent si on identifiait mon conjoint comme le père». (Partenaire d'un donneur)
\end{abstract}

\section{Implication des proches du donneur dans la vie de l'enfant}

Même si les donneurs ne considèrent pas d'un mauvais œil d'être soustraits de la filiation de l'enfant et des responsabilités parentales, plusieurs $d$ 'entre eux sont sensibles au fait que leurs proches, particulièrement leur mère, puissent considérer l'enfant né de leur don comme faisant partie de leur système familial. Certains donneurs ont hésité avant de mettre leurs parents au courant de la situation. De fait, ils étaient extrêmement cons- 
cients du désir de leur mère de devenir grand-mère. Pour cinq d'entre eux, la question était d'autant plus délicate qu'ils étaient soit enfants uniques, soit les premiers de leur fratrie à faire des enfants. D'autres ont plutôt donné leur accord au couple lesbien conditionnellement au fait que leurs parents puissent avoir des contacts avec les enfants issus de leurs dons.

«J'ai dit à mes parents que cela avait été très important pour moi, avant d'accepter de participer au projet des filles, de savoir qu'ils pourraient avoir un rôle auprès de l'enfant. Je savais que ça allait être important pour eux [...]. Je pense que ça peut avoir quelque chose à voir avec les sentiments de mes parents. Ils ne font pas vraiment la séparation entre le biologique et le social et donc, ce serait un petit-fils ou une petite-fille qu'ils n'auraient pas l'occasion de connaître. Je sais que cela leur aurait fait énormément de peine». (Donneur)

\section{Scénarios, responsabilités futures et gestion des conflits}

Quatre donneurs se sont engagés à prendre en charge les enfants advenant le décès des mères. Cela leur importait au point où trois d'entre eux ont insisté pour que cela soit inscrit dans le testament des mères. Pour ces participants, il était tout à fait naturel que l'enfant devienne leur responsabilité si jamais il devait arriver malheur aux mères. Il leur était même inenvisageable que l'enfant soit alors élevé par quelqu'un d'autre qu'eux. En outre, pour les mères concernées, cela allait tout à fait de soi.

«C'était important pour moi que, si par hasard, dans le pire scénario, il se passait quelque chose et que toutes les deux périssent dans un accident de voiture, par exemple, qu'à ce moment-là l'enfant devienne ma responsabilité». (Donneur)

Le lien biologique liant le donneur aux enfants n'est toutefois pas toujours l'élément prédominant par rapport à cette décision, les valeurs humaines et éducatives communes étant aussi considérées dans la réflexion des couples concernés.

«Dans leur testament, advenant qu'il arrive quelque chose aux deux, elles [les mères] ont décidé que ça serait [prénom du donneur] et moi qui nous occuperions des enfants. C'est une grande marque de confiance qu'elles nous ont accordée». (Partenaire d'un donneur)

Bien que les mères, les donneurs et leur partenaire de vie partagent des valeurs communes, cela ne veut pas dire que les donneurs sont en accord avec l'ensemble des décisions que prennent les mères concernant leurs enfants. Tous les hommes considèrent toutefois qu'ils n'ont pas la prérogative d'intervenir advenant un désaccord avec les mères, puisque ce sont elles qui détiennent l'autorité parentale. 
«Ce n'est pas à moi de dire : 'Non, non, faites pas ça. Je vous interdis de faire ça'. Ce n'est pas à moi. Pour moi c'est clair depuis le début que je ne suis pas là pour élever ces enfants-là. Des fois, je constate que moi, je ferais les choses différemment, mais je ne pense pas que je sois en position de déterminer si elles ont raison ou tort, c'est juste que je vois ça différemment». (Donneur)

\title{
Sollicitations éventuelles de l'enfant envers le donneur
}

Lorsqu'ils se projettent dans l'avenir, les donneurs font preuve également d'une réelle préoccupation pour le bien-être de l'enfant. Ainsi, à l'exception d'un seul, tous ouvriraient leur porte à l'enfant si ce dernier en manifestait le besoin, et ce, même si cela leur demandait un certain niveau d'adaptation. Plusieurs en avaient déjà discuté avec leur partenaire de vie et presque tous s'étaient déjà posé la question avant qu'elle ne soit abordée lors des entrevues.

«Ma conjointe et moi on se disait : 'Si jamais elle se rebelle et qu'elle se sauve [de son milieu familial], j'ose espérer que c'est chez nous qu'elle va débarquer'». (Donneur)

Par contre, conformément à la représentation qu'ils ont de leur rôle comme étant situé à la marge du système mère-enfant, s'ils acceptaient d'accueillir l'enfant, cela ne se ferait pas sans que les mères ne soient d'accord. Il est clair dans leur propos que l'accueil de l'enfant ne viserait pas à remplacer sa famille d'origine, mais plutôt à l'aider à combler un besoin, celui d'une quête identitaire ou encore, une prise de recul advenant des difficultés entre lui et ses mères.

\begin{abstract}
«Au moment de l'adolescence, quand ils passeront par la période rebelle, s'ils ont envie de se tourner vers nous, comme oasis ou comme îlot pour prendre un temps d'arrêt de leurs vrais parents, nous serions disposés à les prendre. Mais les filles devront être d'accord avec ça. Et si on les accueille, on va leur expliquer qu'on ne le fait pas parce que je suis leur père, mais parce que nous sommes des adultes dans leur vie et que nous sommes là pour les aider au moment où ils en ont besoin». (Donneur)
\end{abstract}

\section{Discussion et conclusion}

Les motivations des mères lesbiennes rencontrées dans cette étude d'avoir recours à un donneur connu pour fonder leur famille et celles des donneurs de participer au projet parental d'autrui corroborent les constats des autres recherches sur le sujet (Berkowitz, Marsiglio, 2007 ; Kelly, 
2009 ; Norsqvist, 2012 ; Ryan, Berkowitz, 2009; Riggs, 2008 ; Svab, 2007).

En filigrane de la négociation, un aspect incontournable du phénomène réside dans l'encadrement normatif découlant de la Loi instituant l'union civile et les nouvelles règles de filiation qui permet d'assurer la prépondérance de la mère n'ayant pas porté l'enfant sur le donneur pour l'établissement de la filiation. Les discussions sur les attentes peuvent donc se dérouler dans un climat serein puisque les craintes rapportées par les mères non statutaires dans d'autres études ne s'actualisent pas au Québec. Cela facilite aussi le concours de la partenaire de vie du donneur puisqu'elle n'a pas à craindre que son conjoint soit dans l'obligation d'endosser un quelconque rôle parental avec d'autres enfants que ceux nés dans le cadre de leur propre union conjugale. Le contexte juridique québécois offre alors un espace de bon aloi pour discuter de sujets particulièrement délicats tels que, par exemple, les termes d'adresse pour désigner le donneur ou l'implication éventuelle de ses proches dans la vie des enfants issus de ses dons. Malgré les avantages indéniables de la Loi 84 pour sécuriser le lien de filiation unissant les deux mères à leurs enfants, les participantes préfèrent néanmoins miser sur la relation qui les unit au donneur plutôt que de tabler sur une convention légale pour encadrer les rôles et responsabilités de chacun à l'égard des enfants. Elles insistent d'ailleurs fortement sur l'aspect relationnel comme facteur de protection pour d'éventuels conflits avec le donneur.

La procréation apparaît, dans le présent contexte, non pas «assistée par» autrui, mais plutôt «négociée avec» le tiers donneur et, dans certains cas, avec son ou sa partenaire de vie. De fait, les partenaires des donneurs sont des actrices et acteurs incontournables de la dynamique familiale, considérant les facteurs d'influence qui motivent leur implication et leur apport dans la négociation de l'entente de procréation. Contrairement à l'image simpliste dépeignant le don comme un acte isolé, nos résultats démontrent que la participation d'un donneur connu dans le projet parental de mères lesbiennes évoque une trame complexe et évolutive, à la rencontre des facteurs d'influence de chacune des parties impliquées et de considérations relationnelles qui émergent du dialogue. Ce dernier semble être un point d'ancrage qui situe le rôle du donneur en adéquation avec les attentes de chacun et chacune. Les facteurs d'influences et la négociation s'entremêlent pour tisser la trame sur laquelle repose l'engagement du donneur auprès de l'enfant issu de ses dons, mais surtout, de la satisfaction de l'ensemble des personnes concernées quant à l'entente instaurée au départ. De fait, entre les deux temps de mesures, de nombreux changements ont jalonné les trajectoires de vie des mères, des don- 
118 De la procréation «assistée par» autrui à la procréation «négociée avec» autrui : dialogue autour de la place du tiers donneur dans le projet parental de mères lesbiennes au Québec

neurs et de leurs partenaires sans que cela ait une incidence sur la relation entre les protagonistes. Force est de constater que le dialogue initial a permis d'organiser la structure familiale et de circonscrire le rôle du donneur à partir d'une convergence des prises de position des participantes et des participants. Cette clarification des rôles anticipés, des besoins et des intentions fait en sorte que les points de vue des mères, des donneurs et de leur partenaire se rejoignent quant à leur place respective auprès de l'enfant.

Cette étude contient plusieurs limites, la première étant d'être circonscrite au territoire québécois. Étant donné le cadre législatif et social particulier à cette province canadienne qui se distingue des autres contextes géographiques étudiés jusqu'à maintenant, la portée des résultats en est affectée. L'absence apparente de conflits entre les personnes rencontrées ne permet pas non plus de généraliser les résultats à l'ensemble des familles lesboparentales dont les enfants sont nés d'un donneur connu, certaines familles vivant des difficultés ne font vraisemblablement pas partie de notre échantillon. Or, ce constat réaffirme l'importance de la négociation de l'entente de procréation, puisque la présence de conflits aurait corroboré nos résultats sur son importance, de même que celle des facteurs d'influence. Le fait que les enfants soient encore petits peut également expliquer, en partie du moins, l'absence de divergence d'opinions quant à l'actualisation du rôle du donneur. Les enfants étant encore trop jeunes pour être en mesure de réclamer le cas échéant une plus grande implication du donneur dans leur vie, l'orchestration familiale telle que réfléchie et organisée par les adultes est maintenue. Enfin, le niveau de scolarité élevé de nos répondantes et répondants a pu pu influencer le regard posé sur leur situation familiale. De fait, plusieurs femmes et hommes rencontrés étaient au fait de certaines études sur la paternité ou encore, sur les familles homoparentales. Leurs représentations de la paternité ou, encore, leur regard sur la place spécifique du père pour le développement de l'enfant ont pu en être influencés.

Un apport important de cette étude réside dans le fait d'avoir triangulé le point de vue de tous les acteurs concernés. En effet, les recherches portant sur le rôle joué par le donneur au sein des familles lesboparentales documentent les expériences de mères et des donneurs sans nécessairement les mettre en dialogue. En outre, la deuxième collecte de données a permis de valider les hypothèses ayant émergé lors du premier temps de la recherche et confirme l'importance primordiale de la négociation de départ pour le maintien de la relation unissant les mères, les donneurs et leurs partenaires de vie. Une troisième collecte de données est en cours et vise à inclure le point de vue des enfants concernés par ces situations, 
soit ceux que les donneurs ont eu dans le cadre d'une relation conjugale et ceux nés de leurs dons et grandissants dans des familles lesboparentales (Conseil de recherches en sciences humaines du Canada 2014-2016). Cette collecte de donnée permettra de brosser un portrait riche, nuancé et global de la concrétisation de projets parentaux à l'aide d'un tiers donneur, puisque les données recueillies concernent uniquement les points de vue des adultes impliqués.

\section{Références}

Almeling R. (2011), Sex Cells: The Medical Market for Eggs and Sperm, Berkeley, University of California Press.

BARDIN L. (2007), L'analyse de contenu, 11ème édition, Paris, Presses universitaires de France.

Berkowitz D., Marsiglo W. (2007), "Gay Man: Negotiating Procreative, Father, and Family Identities», Journal of Marriage and Family, 69 (2), May, pp. 366-381, https:// doi.org/10.1111/j.1741-3737.2007.00371.x.

BuREAU M.-F. (2009), Le droit de la filiation entre ciel et terre : étude du discours juridique québécois, Cowansville, Éditions Yvon Blais.

CôtÉ I. (2012), «Deux mères, un donneur et des enfants. Motivations des actrices et acteurs quant à l'établissement de la famille lesboparentale avec donneur connu», Nouvelles Pratiques Sociales, 24 (2), pp. 84-101, https://doi.org/10.7202/1016349ar.

CôtÉ I. (2014), «Du père au géniteur en passant par le tiers intéressé : représentations du rôle joué par le donneur de sperme connu dans les familles lesboparentales», Revue Internationale Enfances Familles Générations, 1 (21), pp. 70-95, https:// doi.org/10.7202/1025960ar.

Côté I., LAVoie K., DE Montigny F. (2015), «J'ai aidé deux femmes à fonder leur famille. Le don de gamètes entre particuliers en contexte québécois», Revue Internationale Enfances Familles Générations, 23, pp.127-147, https://doi.org/10.7202/ 1034204ar.

Daniels K., Kramer W., Perez-Y-Perez M. V. (2012), «Semen Donors who Are Open to Contact with their Offspring: Issues and Implications for them and their Families», Reproductive Biomedicine Online, 25, pp. 670-677, https://doi.org/10.1016/j.rbmo. 2012.09.009.

DEMPSEY D. (2010), «Conceiving and Negotiating Reproductive Relationships: Lesbians and Gay Men Forming Families with Children», Sociology, 44 (6), pp. 1'1451'162. 
120 De la procréation «assistée par» autrui à la procréation «négociée avec» autrui : dialogue autour de la place du tiers donneur dans le projet parental de mères lesbiennes au Québec

DEMPSEY D. (2012), «More Like a Donor or More Like a Father? Gay Men's Concept of Relatedness to Children», Sexualities, 15 (2), pp. 156-174, https://doi.org/10. $1177 / 1363460711433735$.

Descoutures V. (2010), Les mères lesbiennes : contribution à une sociologie de la parentalité, Paris, Presses Universitaires de France, https://doi.org/10.3917/puf.desco. 2010.01.

Elder G. H., Johnson M. K., Crosnoe R. (2004), «The Emergence and Development of Life Course Theory", J. T. MoRTIMER, M. J. ShANAHAN (eds), Handbook of the Life Course, New York, Kluwer Academic Publishers, pp. 3-22.

Gouvernement du Québec (2002), Loi instituant l'union civile et les nouvelles règles de filiation, Québec, Assemblée nationale.

Gouvernement du Canada (2004), Loi sur la procréation assistée, Ottawa, Ministère de la Justice.

Gross M. (2015), «L'homoparentalité et la transparentalité au prisme des sciences sociales : révolution ou pluralisation des formes de parenté ?», Enfances Familles Générations, 23, pp. i-xxxvii, https://doi.org/10.7202/1034196ar.

Kelly F. (2009), «(Re)forming Parenthood: The Assignment of Legal Parentage within Planned Lesbian Families», Ottawa Law Review, 40 (2), pp. 185-224.

Mamo L. (2007), Queering Reproduction: Achieving Pregnancy in the Age of Technoscience, Durham, Duke University Press, https://doi.org/10.1215/9780822390220.

Millbank J. (2008), «The Limits of Functional Family: Lesbian Mother Litigation in the Era of the Eternal Biological Family", International Journal of Law, Policy and the Family, 22 (2), pp. 149-177, https://doi.org/10.1093/lawfam/ebn001.

NoRdQVISt P. (2012), «'I don't Want Us to Stand out more than we Already Do': Lesbian Couples Negotiating Family Connections in Donor Conception», Sexualities, 15 (5-6), pp. 644-661, https://doi.org/10.1177/1363460712446271.

NordqVSIT P., SMART C. (2014), Relative Strangers: Family Life, Genes and Donor Conception, Basingstoke, Palgrave Macmillan, 195 p.

PIRES A. (1997), «Échantillonnage et recherche qualitative : essai théorique et méthodologique», J. Poupart, J.-P. Deslauriers, L.-H. GroulX, A. LAPerrière, R. Mayers, A. P. PIRES (eds), La recherche qualitative : enjeux épistémologiques et méthodologiques, Montréal, QC, Gaëtan Morin, pp. 113-169.

RIGGs D. W. (2008), «Lesbian Mothers, Gay Sperm Donors, and Community: Ensuring the Well-Being of Children and Families", Health Sociology Review, 17, pp. 226-234, https://doi.org/10.5172/hesr.451.17.3.226.

Ryan M., Berkowitz D. (2009), "Constructing Gay and Lesbian Families 'Beyond the Closet'», Qualitative Sociology, 32, pp. 153-172, https://doi.org/10.1007/s11133009-9124-6. 
SAvoie-ZAJC L. (2009), "L'entrevue semi-dirigée», B. GAUTHIER (ed), Recherche sociale. De la problématique à la collecte de données, Québec, Les Presses de l'Université du Québec, pp. 337-360.

SURTEes N. (2011), "Family Law in New Zealand: The Benefits and Costs for Gay Men, Lesbians, and their Children", Journal of GLBT Family Studies, 7 (3), pp. 245-263, https://doi.org/10.1080/1550428X.2011.564945.

Svab A. (2007), "New Ways of Parenting: Fatherhood and Parenthood in Lesbian Families», Revija za sociologiju, XXXVIII (1-2), pp. 43-55. 